\title{
Efficacy and toxicity of 8 day methotrexate/folinic acid regimen in the management of low risk gestational trophoblastic neoplasia: a prospective observational study
}

\author{
Murali Subbaiah, Sujithradevi Radhakrishnan*, Jayalakshmi Dorairajan
}

Department of Obstetrics and Gynecology, JIPMER, Puducherry, India

Received: 03 May 2021

Accepted: 29 May 2021

\section{*Correspondence:}

Dr. Sujithradevi Radhakrishnan,

E-mail: sujiradjan@gmail.com

Copyright: (C) the author(s), publisher and licensee Medip Academy. This is an open-access article distributed under the terms of the Creative Commons Attribution Non-Commercial License, which permits unrestricted non-commercial use, distribution, and reproduction in any medium, provided the original work is properly cited.

\section{ABSTRACT}

Background: Gestational trophoblastic neoplasia (GTN) is a rare malignancy that arises from abnormal proliferation of trophoblastic cells. Low risk GTN responds well to single agent chemotherapy with good survival rate. Objective of the study was to assess the efficacy and toxicity of methotrexate chemotherapy in low risk GTN patients receiving 8day methotrexate (MTX)/folinic acid (FA) regimen. A prospective observational study done in a tertiary care hospital in South India.

Methods: This prospective observational study was conducted at a tertiary care hospital in south India between July 2015 and July 2018 and included 56 patients with low risk GTN treated with 8-day MTX/FA regimen.

Results: Among the 56 patients treated, complete remission was achieved in $50(89.3 \%)$. Molar pregnancy was the most common preceding pregnancy event $(96.7 \%)$ and $63.3 \%$ of them developed GTN within 4 months of evacuation. Six non responders were treated with EMACO regimen and achieved complete remission. The most common toxicities were mucositis (46.4\%) and neutropenia (17.8\%). Overall survival of low risk GTN was $100 \%$ at the end of median follow up period of 14.4 months.

Conclusions: For patients with low risk GTN, 8-day MTX/FA regimen is associated with a high rate of remission and low toxicity.

Keywords: Gestational trophoblastic neoplasia, Methotrexate, Toxicity

\section{INTRODUCTION}

Gestational trophoblastic neoplasia (GTN) is a spectrum of pregnancy related tumours arising from abnormal proliferation of trophoblastic cells. ${ }^{1}$ The spectrum ranges from invasive mole, choriocarcinoma, epithelioid tumour and placental site trophoblastic tumour. ${ }^{2}$ Although GTN mostly occurs following molar pregnancy a small proportion can occur after a non-molar pregnancy like miscarriage and term pregnancy. ${ }^{3}$

GTN is classified into low risk and high risk by modified World Health Organisation (WHO) prognostic risk scoring. ${ }^{2}$ GTN is a highly chemo sensitive tumour with a $5 \mathrm{yr}$ survival of $100 \%$ for low risk GTN and $75-90 \%$ for high risk GTN. ${ }^{4,5}$ Low risk GTN is treated with single agent chemotherapy. The most used single agent chemotherapy drugs are Methotrexate (MTX) and Actinomycin-D. ${ }^{6}$ Many centres prefer 8-day MTX/folinic acid (FA) regimen as the first line treatment for low risk GTN due to its high efficacy, easy availability, and low cost. $^{7}$ A recent large retrospective study comparing this regimen with one-day methotrexate infusion regimen reported higher remission rate and lower MTX resistance with the 8 - day MTX regimen. ${ }^{8}$ However, they reported higher treatment-related adverse events with this regimen 
and emphasised the need for prospective studies to study MTX related toxicity. There are several retrospective studies from both developing and developed countries in which MTX toxicity has been studied. ${ }^{9-12}$ However, underreporting of toxicities and non-documentation of minor toxicities are common in such retrospective studies. We did this prospective study to accurately report on the toxicity profile and efficacy of 8-day MTX/FA regimen.

\section{METHODS}

This was a single institute prospective observational study conducted between July 2015 and July 2018 in a tertiary care hospital in south India. Study was conducted after obtaining Institute ethical committee clearance (JIP/IEC/SC/29/886). All patients diagnosed as low risk GTN during the study period were recruited in the study after obtaining informed written consent. GTN was diagnosed according to International Federation of Gynaecology and Obstetrics (FIGO) Gynaecology Oncology Committee guidelines "plateauing of $\beta$-hCG $(\leq 10 \%)$ for four measurements over 3 weeks or a rise in $\beta$ hCG $(>10 \%)$ for three measurements over at least 2 weeks or if there was a histopathological diagnosis of choriocarcinoma". Patients with FIGO stage I-III GTN with modified WHO score $\leq 6$ were categorised as low risk GTN.

Base line demographic characteristics of the patients including age, BMI, FIGO stage and WHO score of GTN were noted. All the patients underwent Investigations like chest $\mathrm{X}$ ray, pelvic and abdominal ultrasound. All the patients also underwent CECT of abdomen and chest. This was done as per the hospital policy although not mandatory according to FIGO guidelines. ${ }^{2}$ The chemotherapy regimen consisted of an 8-day regimen of MTX $(1 \mathrm{mg} / \mathrm{kg}$ on days $1,3,5$ and 7$)$ alternating with FA $(0.1 \mathrm{mg} / \mathrm{kg}$ on days 2, 4, 6 and 8). This regimen was repeated every 2 weeks till serum $\beta$-hCG levels became normal (< 5 IU/l), after which 2 more cycles of the same regimen were given. Serum $\beta$-hCG levels were checked every week till clinical remission, after which the testing was done monthly for 1 year. Complete remission was confirmed when three consecutive serum $\beta$-hCG values were normal (< $5 \mathrm{IU} / \mathrm{l})$.

Haematological investigations including complete blood count (CBC) with platelet count, liver, thyroid and renal function tests were done in all patients before starting chemotherapy. CBC with platelet count, aspartate aminotransferase (AST) levels were repeated before administration of each dose of MTX. In addition, blood urea and serum creatinine levels were also checked before each MTX dose. This was done for accurately documenting toxicities for study purpose. Apart from these blood tests, detailed history was taken, and clinical examination of patients were done before each MTX injection to look for and grade the toxicities/adverse events. Adverse events were graded according to standardized criteria (Common Terminology Criteria for Adverse Events-CTCAE v4.0). During the treatment, any delay in receiving chemotherapy due to toxic effects was also noted.

\section{RESULTS}

Sixty women with low risk GTN were treated with 8-day MTX/FA regimen during the study period. None of the patients underwent repeat curettage or hysterectomy for low risk GTN. Four patients were lost to follow up, hence not included in the analysis. None of the low risk patients received any other regimen as first line therapy for low risk GTN in our centre. The baseline demographic characteristics of the patients are shown in Table 1.

The mean age of the patient was $28.3 \pm 7.1$ years. In 58 $(96.7 \%)$ patients, the antecedent pregnancy event was vesicular mole. The time interval between the antecedent pregnancy event and diagnosis of GTN was less than 4 months in $38(63.3 \%)$ patients. Among these 56-low risk GTN patients, $50(89.3 \%)$ achieved complete remission with the 8-day MTX/FA regimen. The median number of cycles required to achieve complete response was five. One patient with FIGO stage III was included as the risk score was less than 6. Six patients did not respond to this regimen and were treated with EMACO regimen and achieved complete remission. During the follow up period (12 months) there was no recurrence in any of the patients. The overall survival was $100 \%$ at the end of median follow up of 14.4 months.

Table 1: Baseline clinical characteristics of patients with low risk low-risk gestational trophoblastic neoplasia $(n=60)$.

\begin{tabular}{|ll|}
\hline Patient characteristics & Values \\
\hline Age (years) & $18-38$ \\
Mean \pm SD Range & $28.2 \pm 7.1$ \\
\hline Parity, Median (Range) & $2(0-4)$ \\
\hline BMI $\left(\mathrm{kg} / \mathrm{m}^{2}\right)$ Mean \pm SD & $22.3 \pm 2.1$ \\
\hline $\begin{array}{l}\text { Pre- treatment } \beta \text {-hCG } \\
\text { (IU/L) Median range }\end{array}$ & 39,500 \\
\hline Antecedent pregnancy & $(3400-207,091)$ \\
\hline Molar pregnancy, n $(\%)$ & $58(96.7)$ \\
\hline Abortion, $(\%)$ & $2(3.3)$ \\
\hline Time between antecedent & \\
pregnancy and diagnosis, n $(\%)$ & \\
\hline$<4$ months & $38(63.3)$ \\
\hline $4-6$ months & $18(30)$ \\
\hline $7-12$ months & $4(6.7)$ \\
\hline$>12$ months & 0 \\
\hline FIGO staging, $\mathbf{( \% )}$ & \\
\hline Stage - I & $56(93.3)$ \\
\hline Stage -2 & $3(5)$ \\
\hline Stage -3 & $1(1.7)$ \\
\hline FIGO Score, $\mathbf{n}(\%)$ & $34(56.7)$ \\
\hline$\leq 2$ & $18(30)$ \\
\hline $3-4$ & $8(13.3)$ \\
\hline $5-6$ & \\
\hline
\end{tabular}


Table 2: Toxicity of 8-day methotrexate/folinic acid regimen.

\begin{tabular}{|llllll|}
\hline Adverse event & $\mathbf{N}(\%)$ & $\begin{array}{c}\text { Grade-1 } \\
\mathbf{N}(\%)\end{array}$ & $\begin{array}{l}\text { Grade } \mathbf{~} \\
\mathbf{N}(\%)\end{array}$ & $\begin{array}{c}\text { Grade 3 } \\
\mathbf{N}(\%)\end{array}$ & $\begin{array}{l}\text { Grade } 4 \\
\mathbf{N}(\%)\end{array}$ \\
\hline Mucositis & $26(46.4)$ & $20(35.7)$ & $3(5.3)$ & $3(5.3)$ & 0 \\
\hline Liver dysfunction & $4(7.1)$ & $4(7.1)$ & 0 & 0 & 0 \\
\hline Anaemia & $6(10.7)$ & $5(8.9)$ & $1(1.8)$ & 0 & 0 \\
\hline Thrombocytopenia & $5(8.9)$ & $3(5.3)$ & $2(3.6)$ & 0 & 0 \\
\hline Neutropenia & $10(17.8)$ & $3(5.3)$ & 0 & $7(12.5)$ & 0 \\
\hline Renal toxicity & $1(1.8)$ & 0 & 0 & $1(1.8)$ & 0 \\
\hline Nausea & $7(12.5)$ & $7(12.5)$ & 0 & 0 & 0 \\
\hline Vomiting & $1(1.8)$ & $1(1.8)$ & 0 & 0 & 0 \\
\hline Alopecia & $5(8.9)$ & $4(7.1)$ & $1(1.8)$ & 0 & 0 \\
\hline Diarrhoea & $1(1.8)$ & $1(1.8)$ & 0 & 0 & 0 \\
\hline Dry eyes & $4(7.1)$ & $4(7.1)$ & 0 & 0 & 0 \\
\hline Conjunctivitis & $3(5.3)$ & $3(5.3)$ & 0 & 0 & 0 \\
\hline
\end{tabular}

The adverse events and details of toxicity seen with 8-day MTX/FA regimen is given in Table 2. The most common adverse event noted was mucositis, seen in $26(46.4 \%)$ patients. Ten $(17.8 \%)$ patients developed neutropenia, most of which were grade 3 . One of these patients had grade 3 febrile neutropenia. No other infections, pleuritic pain, photosensitivity, or skin rash were documented in any patient. In $7(12.5 \%)$ patients, chemotherapy was delayed because of these toxicities and 3(5.3\%) patients received growth factor to treat neutropenia.

\section{DISCUSSION}

The 8-day MTX/FA regimen achieved complete remission in $89.3 \%$ of patients with low-risk GTN in this study. There were no recurrences and there was $100 \%$ survival at 14.4 months median follow up. The most common toxicities noted were mucositis and neutropenia which were reported in $46.4 \%$ and $17.8 \%$ of patients, respectively. None of the patients had to receive alternate drug regimen because of drug toxicity.

Low risk GTN is very sensitive to single agent chemotherapy. ${ }^{1}$ Treatment of low risk GTN includes a variety of single drug regimens. Most frequently used single drug regimens are weekly MTX, daily MTX for 5 days, 8-day MTX/FA, biweekly actinomycin D and 5 days actinomycin $\mathrm{D}^{2}$ As the literature is insufficient to demonstrate the superiority of one over the other, and each institute has its own preference of drug regimen. A Cochrane database review in 2016 reported that actinomycin $\mathrm{D}$ based regimen may achieve higher primary cure rate compared to MTX for low risk GTN. ${ }^{6}$ However, $55 \%$ of patients from this data base had received weekly MTX regimen. Weekly MTX regimen has been found to have lower efficacy than 8-day MTX/FA regimen and is no longer recommended. ${ }^{13} \mathrm{~A}$ recent trial has reported better complete response rate with multi day MTX regimen compared to actinomycin. ${ }^{14}$ However, this trial was closed prematurely because of slow accrual and the analysis of the report are only considered exploratory.
MTX based regimen are commonly used because of the lower toxicity, ease of administration and storage, and cost-effectiveness. ${ }^{15,16}$ Eight-day MTX/FA is a commonly used regimen worldwide and has been found to have good efficacy and low toxicity. ${ }^{15,17}$ The same dosage can be used for overweight/obese patients with similar efficacy and toxicity. ${ }^{18}$ Mito 9 study compared 2 doses of MTX $(1 \mathrm{mg} / \mathrm{kg} /$ day versus $50 \mathrm{mg} /$ day $)$ in the 8 day MTX/FA regimen and found similar efficacy and toxicity with both doses. ${ }^{17}$

The remission rate in this study was $89.3 \%$ with 8 -day MTX/FA regimen. Similar rates of remission have been reported by other studies using the same regimen for low risk GTN ${ }^{8,10}$ A retrospective study conducted at New England Trophoblastic Disease Centre (NETDC) on 151 patients reported $84.1 \%$ remission rate with 8 -day MTX/FA regimen. ${ }^{8}$ However, a retrospective study from France involving 142 low risk GTN patients reported a lower remission rate of $77.5 \% .{ }^{19} \mathrm{~A}$ retrospective study from India where 25 out of 48 cases of low risk GTN were treated with 8-day MTX/FA regimen achieved 96\% remission rate. ${ }^{9}$ The median number of cycles required for complete remission in our study was 5 ; similar number of cycles were administered in other studies. ${ }^{9}$ Although there were no recurrences in our study, some studies have reported recurrence rates ranging from $1.4 \%$ to $3.6 \%{ }^{8,19}$ Most studies have reported $100 \%$ survival in low risk GTN which was evident in this study also.

Most common toxicity of methotrexate in this study was oral mucositis seen in $46.4 \%$ patients, of which only 3 (5.3\%) patients had grade 3 toxicity. Similar rate of oral mucositis (40\%) was reported in a retrospective study conducted on 151 patients at NETDC. ${ }^{8}$ Another retrospective study from Brazil involving 115 patients who received this regimen reported oral mucositis in $66 \%$ of patients..$^{20}$ However, a retrospective study from India reported mucositis in only $16 \%$ of the patients. ${ }^{9}$ Twentyfive women with low risk GTN received 8day MTX/FA 
regimen in that study. Findings from our prospective study indicate higher rate of mucositis as mentioned already.

Ten $(17.8 \%)$ patients developed neutropenia in our study. This is much lower than that reported from the retrospective study from NETDC in which $27 \%$ of the patients developed neutropenia. ${ }^{8}$ Thirty four of the 37 patients who developed neutropenia in that study had grade 1 or 2 neutropenia. In contrast, 7 of the 10 patients in our study had grade 3 neutropenia. Our findings are similar to findings from a retrospective study from India, in which $12 \%$ of the patients developed neutropenia. ${ }^{9}$ There was delay in chemotherapy in $16 \%$ of the patients because of drug toxicity and $4 \%$ patients received growth factor in that study. This is similar to findings from our study.

Switching to second-line chemotherapy occurred in 5.3\% of the patients due to treatment related grade 3-4 adverse events in the study from NETDC. ${ }^{8}$ Only $1.4 \%$ of patients needed switch over to second line therapy because of MTX toxicity in a study from France. ${ }^{19}$ None of the patients from our study had to switch over to second line chemotherapy because of drug toxicity. Similar findings have been reported from other studies. ${ }^{9,10}$ Only $5.3 \%$ of patients developed conjunctivitis and $7.1 \%$ had dry eye in our study. Some studies have reported $15 \%-33 \%$ rate of dry eyes in these patients. ${ }^{8,20}$ The reason for lower rates in our patients is not known. Other studies from Asia have not reported on the rates of dry eyes and conjunctivitis with this regimen. ${ }^{9,10}$

There is no consensus regarding frequency of doing blood investigations when patients are receiving this regimen. According to the protocol suggested by NETDC; CBC with platelet count and AST levels are to be repeated before administration of each dose of MTX. ${ }^{21}$ However, blood investigations are repeated every 2 weeks in other centres. $^{19}$ At our Centre, the protocol suggested by NETDC is followed.

Merits of this study are the prospective study design, where adverse events were periodically evaluated and accurately quantified by frequent blood investigations and clinical examination. Demerit of the study is the small sample size and that it is a single institute study.

Most studies in the literature about the toxicity of methotrexate in low risk GTN treatment are retrospective and are from developed countries. Higher rates of mucositis and dry eyes were detected in our prospective study compared to retrospective studies from India. The information provided from this prospective study may help in proper counselling of women. Periodic and detailed clinical examination of patients during the chemotherapy may help in early detection of adverse events. Even if the adverse events are minor, their symptomatic treatment and counselling will provide reassurance to the patient.

\section{CONCLUSION}

The 8-day MTX/FA regimen is highly effective in treating low risk GTN with acceptable toxicity.

\section{Funding: No funding sources \\ Conflict of interest: None declared \\ Ethical approval: The study was approved by the Institutional Ethics Committee}

\section{REFERENCES}

1. Seckl MJ, Sebire NJ, Berkowitz RS. Gestational trophoblastic disease. Lancet. 2010;376:717-29.

2. Ngan HYS, Seckl MJ, Berkowitz RS, Xiang Y, Golfier F, Sekharan PK. Update on the diagnosis and management of gestational trophoblastic disease. Int J Gynaecol Obstet. 2018;143(2):79-85.

3. Al-Husaini H, Soudy H, Darwish A, Ahmed M, Eltigani A, Edesa W, et al. Gestational trophoblastic neoplasia: treatment outcomes from a single institutional experience. Clin Transl Oncol. 2015;17:409-15.

4. McNeish IA, Strickland S, Holden L, Rustin GJS, Foskett M, Seckl MJ, et al. Low-risk persistent gestational trophoblastic disease: outcome after initial treatment with low-dose methotrexate and folinic acid from 1992 to 2000. J Clin Oncol. 2002;20:1838-44.

5. Bower M, Newlands ES, Holden L, Short D, Brock C, Rustin GJ, et al. EMA/CO for high-risk gestational trophoblastic tumors: results from a cohort of 272 patients. J Clin Oncol. 1997;15:2636-43.

6. Lawrie TA, Alazzam M, Tidy J, Hancock BW, Osborne R. First-line chemotherapy in low-risk gestational trophoblastic neoplasia. Cochrane Database Syst Rev. 2016;2016(6):CD007102.

7. Goldstein DP, Berkowitz RS, Horowitz NS. Optimal management of low-risk gestational trophoblastic neoplasia. Expert Rev Anticancer Ther. 2015;15:1293-304.

8. Maestá I, Nitecki R, Horowitz NS, Goldstein DP, Moreira FSM, Elias KM, et al. Effectiveness and toxicity of first-line methotrexate chemotherapy in low-risk postmolar gestational trophoblastic neoplasia: The New England Trophoblastic Disease Center experience. Gynecol Oncol. 2018;148:161-7.

9. Apia GS, Gupta JS, Maheshwari A, Deodhar K, Kerkar RA, et al. Outcome of gestational trophoblastic neoplasia: Experience from a tertiary cancer centre in India. Clin Oncol. 2014;26:39-44.

10. Anfinan N, Sait K, Sait H. Gestational trophoblastic disease in the western region of Saudi Arabia (singleinstitute experience). Eur J Obstet Gynecol Reprod Biol. 2014;180:8-11.

11. Khan F, Everard J, Ahmed S, Coleman RE, Aitken M, Hancock BW. Low-risk persistent gestational trophoblastic disease treated with low-dose methotrexate: efficacy, acute and long-term effects. Br J Cancer. 2003;89(12):2197-201. 
12. Shrivastava S, Katia AC, Barmon D, Deka P, Bhuyan C, Bhargav SJ. Gestational trophoblastic neoplasia: A 6 year retrospective study. South Asian J Cancer 2014; 3:33-7.

13. Abu-Rustum NR, Yashar CM, Bean S, Bradly K, Campos SM, Chon HS, et al. Gestational Trophoblastic Neoplasia, Version 2.2019, NCCN Clinical Practice Guidelines in Oncology. J Natl Compr Cano Netw 2019; 17:1374-91.

14. Schink JC, Filiaci V, Huang HQ, Tidy J, Winter M, Carter J, et al. An international randomized phase III trial of pulse actinomycin-D versus multi-day methotrexate for the treatment of low risk gestational trophoblastic neoplasia; NRG/GOG 275. Gynecol Oncol 2020; 158:354-60.

15. Brown J, Naumann RW, Seckl MJ, Schink J. 15years of progress in gestational trophoblastic disease: Scoring, standardization, and salvage. Gynecol Oncol 2017; 144:200207.

16. Miller CR, Chappell NP, Sledge C, Leath CA, Phippen NT, Havrilesky LJ, et al. Are different methotrexate regimens as first line therapy for low risk gestational trophoblastic neoplasia more cost effective than the dactinomycin regimen used in GOG 0174? Gynecol Oncol 2017; 144:125-129.

17. Mangili G, Cioffi R, Danese S, Frigerio L, Ferrandina G, Cormio G, et al. Does methotrexate (MTX) dosing in a 8-day MTX/FA regimen for the treatment of low- risk gestational trophoblastic neoplasia affect outcomes? The MITO-9 study. Gynecol Oncol. 2018;151:449-52.

18. Maestá I, Horowitz NS, Goldstein DP, Bernstein MR, Ramírez LA, Moulder J, et al. Response to chemotherapy in overweight/obese patients with lowrisk gestational trophoblastic neoplasia. Int J Gynecol Cancer. 2015;25:734-40.

19. Chalouhi GE, Golfier F, Soignon P, Massardier J, Guastalla JP, Trillet-Lenoir V, et al. Methotrexate for 2000 FIGO low-risk gestational trophoblastic neoplasia patients: efficacy and toxicity. Am J Obstet Gynecol. 2009;200:643.

20. Uberti EM, Fajardo M, Cunha AG, Frota SS, Braga A, Ayub AC. Treatment of low risk gestational trophoblastic neoplasia comparing biweekly eightday Methotrexate with folinic acid versus bolus-dose Actinomycin-D, among Brazilian women. Rev Bras Ginecol Obstet. 2015;37:258-65.

21. Berkowitz RS, Goldstein DP, Bernstein MR. Ten years' experience with methotrexate and folinic acid as primary therapy for gestational trophoblastic disease. Gynecol Oncol. 1986;23:111-8.

Cite this article as: Subbaiah M, Radhakrishnan S, Dorairajan J. Efficacy and toxicity of 8 day methotrexate/folinic acid regimen in the management of low risk gestational trophoblastic neoplasia: a prospective observational study. Int J Reprod Contracept Obstet Gynecol 2021;10:2744-8. 